\title{
Infinite Measure: Learning to Design in Geometric Harmony with Art, Architecture and Nature by Rachel Fletcher
}

\section{Sylvie Duvernoy}

Published online: 19 March 2015

(C) Kim Williams Books, Turin 2015

\begin{abstract}
Sylvie Duvernoy reviews the book Infinite Measure: Learning to Design in Geometric Harmony with Art, Architecture and Nature by Rachel Fletcher.
\end{abstract}

Keywords Geometry - Geometrical patterns · Dynamic symmetry ·

Proportion · Harmony

Who today still knows how to draw on a sheet of paper a perpendicular to a given line from a given external point with the help of only a compass and a nongraduated ruler? Or how to draw a parallel to a given line from a given external point... again, only with compass and straightedge? Answers to these queries are given respectively on page 104 and 227 of the book by Rachel Fletcher entitled Infinite Measure: Learning to Design in Geometric Harmony with Art, Architecture and Nature (George F. Thompson Publishing, Stauton, VA, USA, 2013) (Fig. 1).

This book is about traditional knowledge in graphic geometry and it revives an ancient heritage: hand drawing as a method to represent geometric magnitudes, compare them, calculate proportional ratios, define proportions, design diagrams, and so forth. This knowledge goes as far back as ancient Greece, when magnitudes were "figurate quantities", represented either by lines, areas or volumes. Arithmetical operations were then achieved through the drawing of figures. When numbers were lines, addition meant lengthening an initial line; multiplication between two numbers meant drawing a rectangle, or constructing a parallelepiped when three numbers were involved. For instance, multiplying a number by itself created a square, and multiplying it once again created a cube. The graphic

\footnotetext{
S. Duvernoy $(\bowtie)$

via Benozzo Gozzoli 26, 50124 Florence, Italy

e-mail: syld@kimwilliamsbooks.com
} 


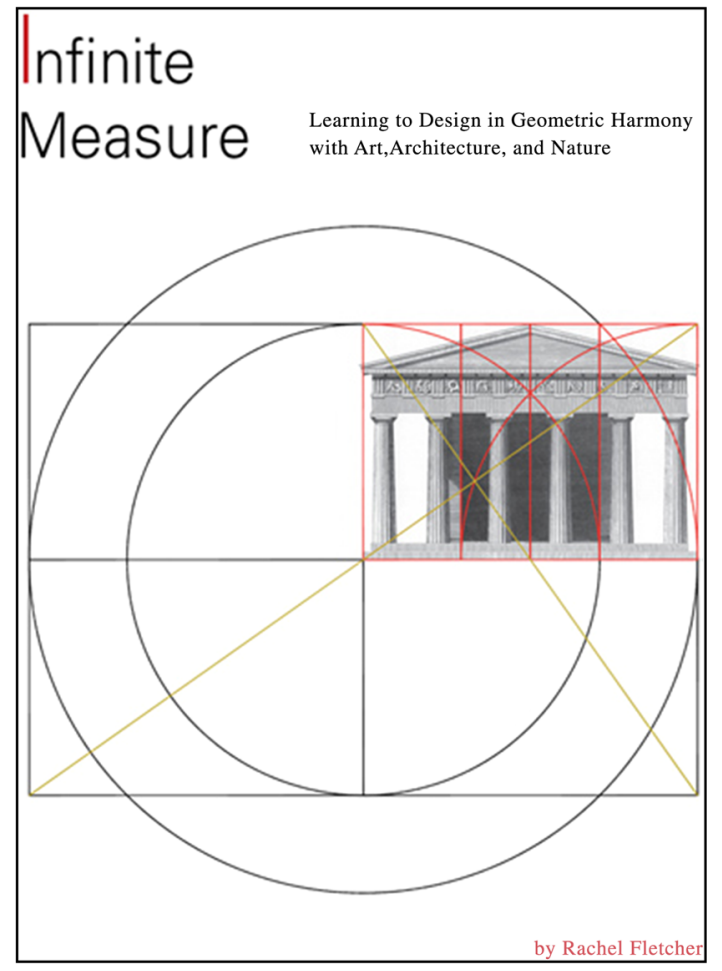

Fig. 1 Cover of Infinite Measure

representation of mathematical objects also made it possible to visually materialise the irrational quantities in addition to the natural integers. They could thus be inserted and manipulated together with rational numbers in research and/or design processes. Plato claimed that just two tools sufficed to solve any problem: the compass for measuring and the straightedge for drawing. We know that at a later date several Greek mathematicians invented other tools to draw special curves that would help solve classical problems such as the duplication of the cube or the trisection of any angle, but those are not addressed by Fletcher, who focuses only on circles and polygons. In fact, this is not a book about the history of geometry but rather a workbook which gathers a selection of traditional geometric tricks for the design trade, without precise historical references. Neither is this a discussion on geometric harmony, even though these two words appear in the subtitle of the book. Although we are not told which patterns are more harmonious and why, the underlying assumption throughout the book's nine chapters is that geometrical order is a road to reach harmony.

Rachel Fletcher is a lighting and stage designer for the theatre, and a self-taught geometer. In her introduction, she explains: "I came to design through the medium of theatre, where the designer's canvas is the world of the stage... I came to understand that basic shapes, the very triangles, squares and pentagons we play with as children, are building blocks for arranging spaces harmonically" (p. 11). For the 
last ten years Fletcher has been the contributing editor of the Nexus Network Journal for the "Geometer's Angle" column, and this book collects most of the work that has been published in the pages of the journal: simple, useful step-by-step procedures to construct and analyze geometric figures to create layouts and diagrams. The book is divided in two parts: (I) Geometry's shapes and symbols, and (II) Composing space plans. Part II is strongly influenced by Jay Hambidge's concept of Dynamic Symmetry. While Hambidge, in his writings, remains purely theoretical, Fletcher gives some examples of application at the end of each chapter.

The author dedicates her book to a quite broad readership: "students, artists, and designers of all visual disciplines and professions, from architecture, interiors, and landscape to painting, sculpture and graphic arts" (p.12). But since the book addresses the issues of planar geometry only, and in 2D geometry only polygons and circles, it might be considered somewhat incomplete and elementary to modern architects and students in architecture. The most complex shape that is inquired in the book is the golden spiral. There is nothing about conics, solids of any kind, not even the sphere and the cube, and the Platonic solids are only mentioned in passing.

However the book will surely be appreciated by those architects and architecture historians interested in the relationship between architecture and mathematics, whose research is devoted to the study of geometrical diagrams and patterns of architecture of the past. We know from the historical literature that architects relied on chosen numbers, proportional ratios and chosen shapes to impart beauty and harmony to their projects, because the mathematicians themselves considered these numbers, ratios and shapes to be beautiful, meaningful and symbolic. Nevertheless architectural treatises hardly give any practical indications on how to manipulate these mathematical objects in order to achieve the fixed goals. The book Infinite Measure unveils many clues to the process of creating a geometrical pattern. The multiple constructions, the number of variations, the creativity, the infinite possibilities, will inspire researchers and, hopefully, designers. The pages of the "Geometer's angle" by Rachel Fletcher of the Nexus Network Journal were always and continue to be very popular among $N N J$ readers. Those who enjoyed the articles will find much more here to learn from. 